\title{
SENSORI-MOTOR RECOVERY IN POST-STROKE SHOULDER SUBLUXATION: A NON INVASIVE CORRELATION STUDY
}

Ushnish Mukherjee ${ }^{1}$, Swapan Kumar Mishra *2, Prabir Mandal ${ }^{3}$, Subhra Mandal ${ }^{4}$, Sourav Kundu ${ }^{5}$, Pankaj Kumar Mandal ${ }^{6}$.

${ }^{1}$ Senior Medical Officer GR-III, Department of Physical Medicine \& Rehabilitation(PM\&R), MR Bangur SSH, Kolkata-33, West Bengal(WB), India.

${ }^{* 2}$ Associate Professor, Department of PM\&R, North Bengal Medical College, WB, India.

${ }^{3}$ Senior Medical Officer GR-I, Department of PM\&R, MR Bangur SSH, Kolkata, WB, India.

${ }^{4}$ Professor \& HOD, Department of Anatomy, Diamond Harbour Medical College, WB, India.

${ }^{5}$ DTO, Paschim Bardhaman, Department of Health \& Family Welfare, Swasthya Bhawan, WB, India.

${ }^{6}$ Professor \& HOD, Department of PM\&R, R G Kar Medical College \& Hospital, Kolkata, WB, India.

\section{ABSTRACT}

Background: Stroke is the most common neurological condition causing long term disabilities in various ways. Post-stroke shoulder subluxation, mainly the Gleno-humeral Subluxation (GHS) of the affected side is often detected as a complication. Numerous theories exist to explain the pathomechanics of developing GHS,but studies regarding its relationship with the sensori-motor recovery of the affected limb is still controversial.

Aim of Study: To ascertain the correlation between Gleno-humeral subluxation with sensori-motor recovery of the affected upper limb following stroke.

Method: This correlation study was conducted on 30 patients of both sexes within the age group of 45-65 years with post-stroke (duration < 6weeks) Gleno-humeral subluxation( GHS). Screening of GHS was done by palpation and degree of GHS was quantified by High resolution USG by measuring the Acromion - Greater Tuberosity Distance difference (AGTDD) between two shoulders. Sensorimotor recovey of the affected limb was assessed by Fugl - Meyer Assessment Scale of Upper Extremity (FMA-UE). Data collected at the baseline (visit1), at 6 weeks (visit 2), 12 weeks (visit 3) and at the end of the study i.e 24 weeks (visit 4).

Results: Pearson Correlation ( $r$ ) in all visits (V1: $r=-0.926, p=0.000$. V2: $r=-0.947, p=0.000$. V3: $r=-0.912, p=0.000$. V4: $r=-0.924, p=0.000$ ) established a statistically significant negative correlation between degree of shoulder subluxation and sensory-motor recovery.

Conclusion: Post-stroke GHS is a significant complication which undoubtedly plays a cardinal negative role during sensori-motor recovery of the affected upper limb.

KEY WORDS: Gleno-humeral subluxation (GHS), Acromion-Greater Tuberosity Distance difference (AGTDD), Fugl-Meyer Assessment Scale of Upper Extremity (FMA-UE).

Corresponding Author: : Dr. Swapan Kumar Mishra, Associate Professor,Department of PM\&R, North Bengal Medical College, Sushrutanagar, Darjeeling, India. phone-8585046797.

E-Mail: docpmr@yahoo.com

Access this Article online

Quick Response code

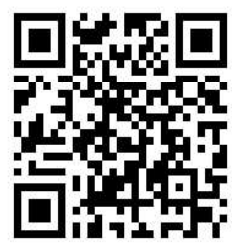

DOI: $10.16965 /$ ijar.2020.119
Journal Information

International Journal of Anatomy and Research

RG Journal ISSN (E) 2321-4287 | ISSN (P) 2321-8967

https://www.ijmhr.org/ijar.htm

DOI-Prefix: https://dx.doi.org/10.16965/ijar

(c) $\mathrm{Br}-\mathrm{MB}-\mathrm{SH}$

Article Information

Received: 05 Feb 2020

Peer Review: 06 Feb 2020

Revised: None
Accepted: 02 Mar 2020

Published (O): 05 Apr 2020

Published (P): 05 Apr 2020 


\section{INTRODUCTION}

Stroke is the most common neurological condition causing long term disabilities in many ways and has extensive emotional and socio-economic consequences [1]. Post-stroke shoulder subluxation, mainly the Gleno-humeral Subluxation (GHS) is a frequent complication present in 17 to $81 \%$ cases [2] and usually seen within three weeks after stroke. ${ }^{3}$ GHS is described as increased translation of the humeral head relative to the glenoid fossa [3]. There are many theories to explain the pathomechanics of developing GHS like, during the initial flaccid period, due to downward pull by the weight of the affected arm, the overstretched rotator cuff cannot perform its function of maintaining the humeral head in the glenoid fossa, leading to the GHS [4]. But according to Basmajian and Bazant [5], during the flaccid stage, the trunk tends to incline or shorten towards the hemiplegic side, which causes the descent of scapula from its normal horizontal level. The trapezius and the serratus anterior also become flaccid, causing the rotating scapula to descend downwards. Without normal tone, the rotator cuff can no longer maintain the integrity of the Gleno humeral Joint (GHJ), leading to GHS. Typically, GHS has been described as Inferior subluxation [2,6], but overall Antero-inferior subluxation is most common $[2,6]$. During the spastic stage, the pectoralis major and minor, rhomboids, levator scapulae, and latissimus dorsi can become hypertonic, further rotating the scapula downward, causing GHS[7].

GHS can be evaluated by various clinical and imaging methods [3]. Clinical evaluation by palpation of the space between the acromion and the head of the humerus to measure GHS has shown to have higher reliability as compared to other clinical methods [7].

But the technique is too much physician dependant. In the last few years, instead of conventional serial radioghaphs ( $x$-ray), recent studies recommended high resolution ultrasonography (HR USG) as a valid and reliable tridimensional diagnostic tool for the measurement of poststroke GHS [8]. Modern researchers are preferring HR USG because it's not only inexpensive and convenient but also radiation free and dynamic technique.

Several studies in the last few decades with contradictory conclusions have pointed GHS as an important risk factor for generating various other symptoms in the affected upper limb like pain, decreased range of motion, difficulties in activities of daily living etc., But till date, controversy still exists regarding the direct correlation between the sensorimotor recovery and the degree of GHS in the hemiparetic shoulder.

Therefore, this correlation study was done by assessing the sensorimotor recovery of the affected limb with Fugl-Meyer AssessmentUpper Extremity [9] (FMA-UE) score and the degree of GHS quantified by HR-USG $[8,10]$.

\section{MATERIALS AND METHODS}

This Correlation Study was initiated after receiving the approval from the institutional ethical committee. All the stroke patients receiving standardized medical and rehabilitation measures from June 2015 to August 2016 at the Department of Physical Medicine \& Rehabilitation, R.G.Kar Medical College \& Hospital, Kolkata ,West Bengal, were screened according to the following inclusion and exclusion criterias and data collected at the baseline (visit1), at 6 weeks (visit 2),12 weeks (visit 3) and at the end of the study i.e 24 weeks (visit 4).

Inclusion criteria were- i). First time stroke patients with post stroke hemiparetic shoulder [2], ii). Stable neurologic status [2], iii). Sufficient communicative ability [1], iv). Age between 45 years to 65 years, v). Duration of stroke $\leq 6$ weeks. vi). Clinical screening of affected shoulder showing palpable gap between the acromion and the humeral head supported by imaging $[1,2,8,3]$.

Exclusion criteria: were- i). Significant pain, restricting hemiparetic shoulder movements. ii). Prior shoulder disorder, surgery, bony pathology impairing the movement of shoulder joints. iii). Poor trunk control that prohibited the maintenance of an upright sitting posture. iv). Bilateral shoulder involvement. v). Upper limb spasticity score $\geq 3$ in Modified Ashworth Scale. vi). Presence of central pain ,Complex Regional 
pain Syndrome, contractures or Hemineglect in affected upper limb. vii). Presence of cerebellar involvement. viii). Patients with diagnosed Brachial plexus injury.

\section{Procedures for measuring parameters:}

GHS measurement $[8,10]$ : When a palpable gap was found clinically(PIC-1), baseline X-ray of both the shoulders (both AP and Lateral view) were done to rule out any bony pathology around shoulder girdles. Thereafter, Ultrasonographic evaluation of Acromion-Greater Tuberosity Distance (AGTD), (PIC-2) and Humero-Glenoid Distance (HGD) were done by using a $8-14 \mathrm{MHz}$ (high resolution) linear probe in both shoulders to quantify the inferior and anterior or posterior GHS respectively. Both were measured in a neutral shoulder position while the patient was sitting upright without any back support and arms hanging by the side of the body. In the superior-inferior direction, the AGTD difference (AGTDD) was measured using a superior Ultrasound Imaging (USI) view. In the anterior-posterior direction, the HGD was measured using a posterior transverse view. Clinically significant difference of distance $\geq$ $5 \mathrm{~mm}^{11}$ between the normal and affected shoulder were recorded in study proforma which was found only in AGTDD measurements.

Sensori-Motor recovery assessment of the whole kinetic chain of the affected upper limb was done by Fugl-Meyer Assessment-Upper Extremity [9] (FMA-UE) with the following testing protocoli). Both verbal as well as nonverbal clear and accurate instructions for the required movements were given. ii). First the patient had to perform the required movements on the non-paretic side. Testing of the paretic side took place when the patient fully understood the required movement. The test was repeated three times and the highest score was written down. iii). Only Verbal encouragements or corrections were done without any manual facilitation. iv). When the performance of the test-item is unclear, the lowest score was written down. v). The passive range of motion (PROM) was used as a reference for determining the active range of motion (AROM). vi). Testing the upper extremity is done while the patient is in the upright (seated) position. Data from above procedures along with independent variables like age, gender, Body Mass Index (BMI), handedness, side involved, type and duration of stroke, care-giver status and presence of co-morbidities were put on pre-designed pre-tested study proforma for statistical analysis on SPSS20.

\section{Study design flow-chart}

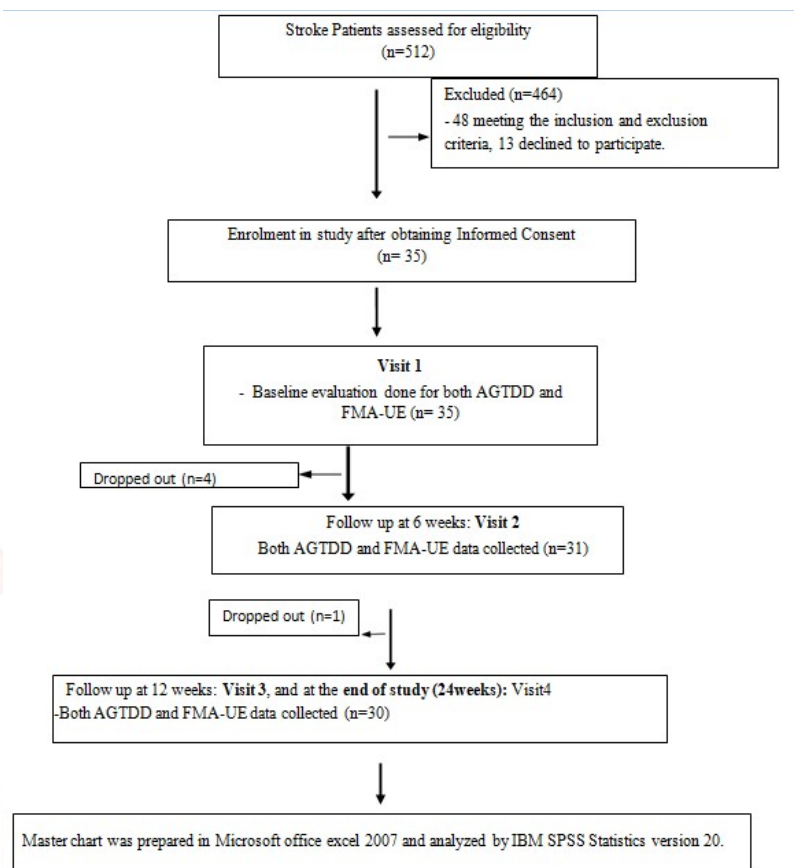

\section{STATISTICAL ANALYSIS AND RESULTS}

The total number of participants in this study were initially 35 . Among them 5 participants were excluded from the analysis as they did not appear for timely follow-ups. So the analysis was done for total 30 participants. Master chart was prepared in Microsoft office excel 2007 and analysed by IBM SPSS Statistics version 20 . Variables were tested for normal distribution by Shapiro-wilk test. All the variables were normally distributed. Correlation between two variables was done by Pearson Correlation. Confidence Interval $(\mathrm{Cl})$ was taken as $95 \%$ and $p$ value $<0.05$ was considered as statistically significant.

Demographical characteristics- The minimum and maximum age among all the patients were 52 years and 65years respectively with mean and standard deviation 59.73 \pm 3.19 (years). Gender distribution showed a male predominance with total $21(70 \%)$ male and $9(30 \%)$ female. Body Mass Index (BMI) of the participants ranged from $17.93 \mathrm{~kg} / \mathrm{m} 2$ to $31.68 \mathrm{~kg} / \mathrm{m} 2$. The mean and standard deviations were $23.17 \pm 3.61 \mathrm{~kg} / \mathrm{m}^{2}$. All the participants in the study were right handed. Due to stroke, right 
upper limb was affected in total 23(76.67\%) patients.

History of smoking was present in 7 patients and history of alcohol intake in 2 patients. But all of them abstained for more than 10 years.

Type of stroke was mainly ischemic affecting total 23(76.67\%) patients, middle cerebral artery territory with most common involvement of lenticulostriate branch. Time gap between the onset of stroke and initiation of this study was $24.83 \pm 5.5$ (days) among all the patients with a range of 16-35days.

Table 1 showing the Range and Mean+Standard Deviation of both GHS (as AGT distance difference) and FMA-UE in different visits with graphical presentation at figure 1 and 2 . Table 2 shows in all visits statistically significant negative co-relation was found between degree of GHS (quantitatively measured as AGTDD) and sensory-motor recovery ( FMA-UE).

Fig. 1: Acromion-Greater tuberosity distance measurement by High resolution USG.

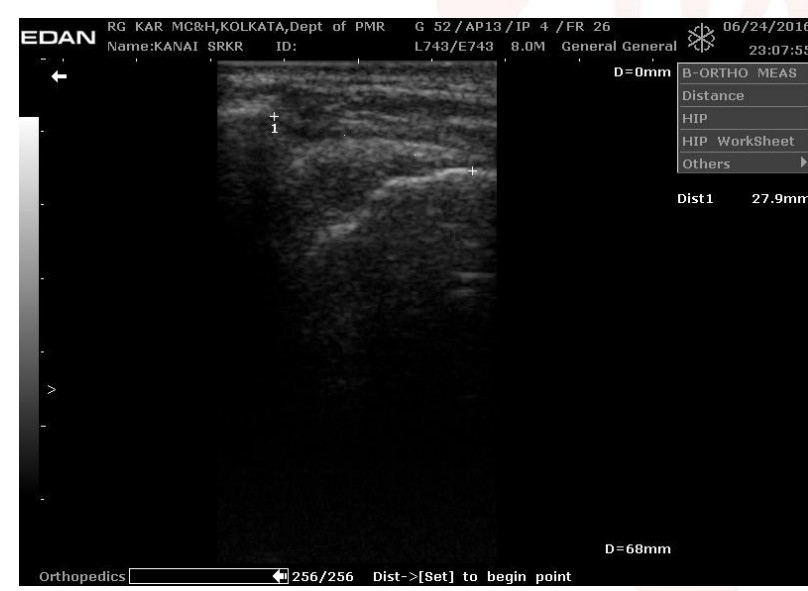

Fig. 2: Screening for detecting shoulder.

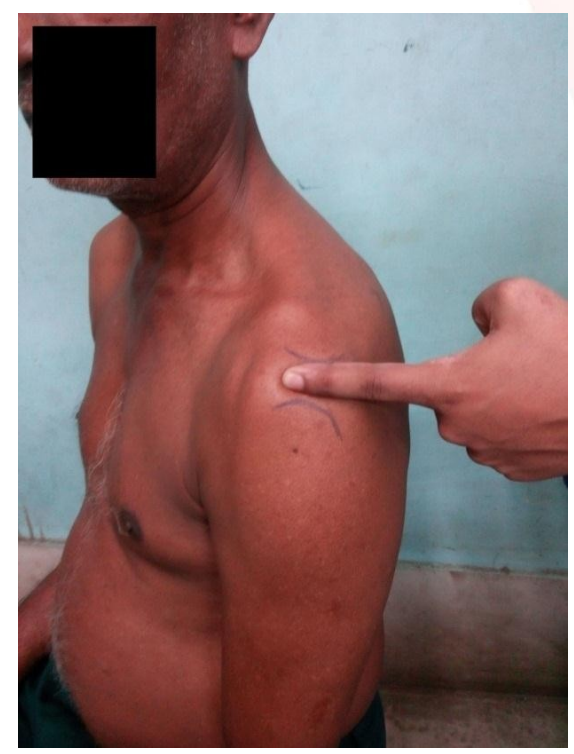

Table 1: Acromion-greater tuberosity distance difference (agtdd) and fma-ue in different visits.

\begin{tabular}{|c|c|c|}
\hline VISIT & AGTDD (mm) & FMA-UE \\
\hline $\begin{array}{c}\text { BASELINE (VISIT 1) } \\
\text { MEAN } \pm \text { SD } \\
\text { RANGE }\end{array}$ & $\begin{array}{c}11.10 \pm 2.07 \\
08-15\end{array}$ & $\begin{array}{c}33.90 \pm 3.26 \\
28-41\end{array}$ \\
\hline FOLLOW UP (VISIT 2) & & \\
MEAN \pm SD & $8.27 \pm 1.76$ & $52.50 \pm 5.17$ \\
RANGE & $06-12$ & $40-61$ \\
\hline FOLLOW UP (VISIT 3) & & \\
MEAN \pm SD & $6.03 \pm 1.40$ & $67.03 \pm 5.29$ \\
RANGE & $04-09$ & $57-79$ \\
\hline STUDY END (VISIT 4) & & \\
MEAN \pm SD & $3.90 \pm 1.24$ & $86.80 \pm 5.82$ \\
RANGE & $02-07$ & $74-96$ \\
\hline
\end{tabular}

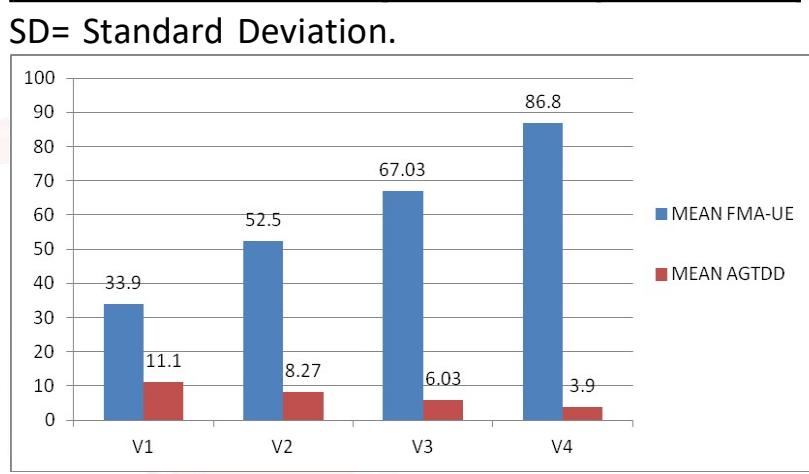

Table 2: Co-relation between degree of shoulder subluxation and sensorimotor recovery.

\begin{tabular}{|c|c|c|c|c|}
\hline \multirow[b]{2}{*}{ FMA-UE } & \multicolumn{4}{|c|}{ AGT DISTANCE DIFFERENCE } \\
\hline & $\begin{array}{c}\text { VISIT1 } \\
\text { (AGTDD }\end{array}$ & $\begin{array}{c}\text { VISIT2 } \\
\text { (AGTDD }\end{array}$ & $\begin{array}{c}\text { VISIT3 } \\
\text { (AGTDD }\end{array}$ & $\begin{array}{c}\text { VISIT4 } \\
\text { (AGTDD }\end{array}$ \\
\hline $\begin{array}{c}\text { VISIT1 } \\
\text { (FMA-UE V1) }\end{array}$ & $\begin{array}{c}n=30 \\
r=-0.926 \\
p=0.000\end{array}$ & & & \\
\hline $\begin{array}{c}\text { VISIT2 } \\
\text { (FMA-UE V2) }\end{array}$ & & $\begin{array}{c}n=30 \\
r=-0.947 \\
p=0.000\end{array}$ & & \\
\hline $\begin{array}{c}\text { VISIT3 } \\
\text { (FMA-UE V3) }\end{array}$ & & & $\begin{array}{c}n=30 \\
r=-0.912 \\
p=0.000\end{array}$ & \\
\hline $\begin{array}{c}\text { VISIT4 } \\
\text { (FMA-UE V4) }\end{array}$ & & & & $\begin{array}{c}n=30 \\
r=-0.924 \\
p=0.000\end{array}$ \\
\hline
\end{tabular}

$r=$ Pearson correlation

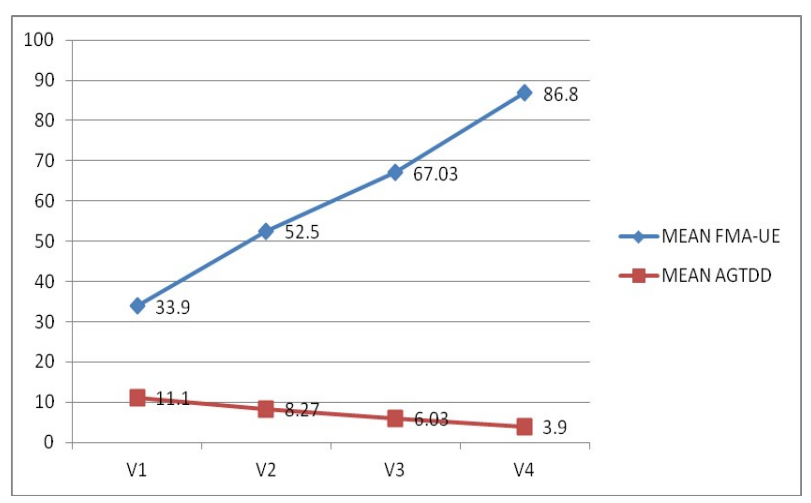




\section{DISCUSSION}

Pathomechanics of Glenohumeral subluxation (GHS) following stroke in the affected limb has several theories in different stages of stroke recovery. It is a frequent complication which is also a controversial independent risk factor for several other post stroke complications. So our aim was to establish a distinct correlation between GHS and recovery of affected limb.

Fugl-Meyer motor and sensory assessment (FMA), developed from the original stages of motor recovery described by Brunnstrom [12], is a standardized method to assess sensorimotor recovery in both upper and lower limbs with high inter-rater reliability. As at the same time, upper and lower extremity in a stroke patient, often stay in a different stages of Brunnstrom recovery, Fugl-Meyer AssessmentUpper Extremity (FMA-UE) [9] was introduced later on to assess only the upper limb functions. The maximum possible total score of FMA-UE is 126. The clinical value of the FMA-UE is that it provides a hierarchical scale of motor impairment severity. So, our study was designed to assess the correlation of FMA-UE with the degree of GHS.

We used HR USG for quantification of GHS as it is well validated and has several advantages. It can easily be performed at the bedside, can simultaneously measure the degree of both inferior and antero-posterior shoulder subluxation by comparing the Acromion-greater tuberosity distance (AGTD) difference and Humero-Glenoid Distance (HGD) respectively between the sound and affected shoulders. All visits showed HGD was $<5 \mathrm{~mm}$ in all patients suggesting all of them were suffering from inferior suluxation and statistical analysis was done as per the Acromion-Greater Tuberosity Distance Difference (AGTDD) in $\mathrm{mm}$.

In our study, at the base level, the maximum AGTDD among the stroke patients was $15 \mathrm{~mm}$ and the minimum was $8 \mathrm{~mm}$, with the mean $\pm S D=11.1 \pm 2.07$. Baseline FMA-UE score ranges from only 28 to 41 out of total possible score of 126 , with mean $\pm S D=33.9 \pm 3.26$. Subsequent visits showed steady increase in FMA-UE score (mean \pm SD at V2: $52.5 \pm 5.17$ \& V3: $67.03 \pm 5.29$ ) suggesting improvement in limb function with simultaneous decreasing pattern in AGTDD (mean \pm SD at V2: $8.27 \pm 1.76 \&$ V3: $6.03 \pm 1.4)$ suggesting decreasing GHS. At the end of the study (visit4), FMA-UE score was maximum of all visits (mean $\pm S D=86.8 \pm 5.82$ ) and AGTDD (mean $\pm S D=3.9 \pm 1.24$ ) was minimum of all visits. When Pearson Correlation was done between these two variables (AGTDD and GHS) statistically significant negative co-relation were found across the visits ( $r$ in visit1 $=-0.926, r$ in visit2 $=-0.947, r$ in visit $3=-0.912, r$ in visit $4=-0.924, p=0.000$ in all visits). This correlation between GHS and sensorimotor recovery clearly stated that decrease in GHS is a factor closely associated with affected arm recovery.

Paci M et al, 2007 [13], in the study- "Shoulder subluxation after stroke: relationships with pain and motor recovery" also concluded that GHS is a factor associated with shoulder pain development and with arm motor recovery and should be treated in the acute stage of stroke.

Although Zorowitz et al.1995 [14] found no correlation between Fugl-Meyer scores and vertical subluxation, but 6 years later, in their study- "Recovery patterns of shoulder subluxation after stroke" Zorowitz et al.2001 [15] found that motor recovery can influence the reduction of GHS. Kumar P et al,2019 [16] concluded-as presence of GHS is related to reduction in shoulder ROM, muscle strength and upper limb function, proper preventive measures should be taken to prevent and or manage GHS, and here lies the clinical implication of our study. With the negative correlation derived from our study it can be strongly recommended that the measures like proper positioning-handling of the affected limb for prevention, along with functional electrical stimulations for managing GHS should be applied to hasten the upper limb recovery, also supported by the studies of Türkkan C et al.2017 [3], Jung KM et al.2019 [17], and meta-analysis by Nadler M, Pauls MM in 2017 [18] and also by Arya KN et al.2018 [19]. Therefore, to conclude, this study showed that the degree of GHS is strongly associated and can hamper the sensory-motor recovery of the affected limb following stroke and proper handling and rehabilitational measures should be planned in early days of stroke for preferably preventing or managing GHS. 
Limitations of the study: Sample size was small. Outcome difference between dominant and non-dominant hemisphere involvement, ischemic and hemorrhagic stroke was not compared. All these limitations are future perspective of our study where dfferent interventional methods for managing GHS can be compared on large scale in different stroke patterns.

\section{Conflicts of Interests: None}

\section{REFERENCES}

[1]. Stein J, Brandstater ME. Stroke Rehabilitation. In: Frontera WR, Delisa JA(eds), DeLisa's Physical Medicine \& Rehabilitation Principle And Practice, 5th ed, Philadelphia PA,USA: Lipincott Williams \& Wilkins a Wolters Kluwer business, 2010; chapter23,p551-571.

[2]. Paci M., Nannetti L, Rinaldi L A; Glenohumeral subluxation in hemiplegia: An overview. Journal of rehabilitation research and development, 2005;42(4):557-68.

[3]. Türkkan C, Öztürk GT, Uðurlu FG, Ersöz M. Ultrasonographic assessment of neuromuscular electrical stimulation efficacy on glenohumeral subluxation in patients with hemiplegia: a randomized-controlled study. Turkish Journal of Physical Medicine and Rehabilitation. 2017 Dec;63(4):287.

[4]. Chaco, J., \& Wolf, E. Subluxation of the glenohumeral joint in hemiplegia. American journal of physical medicine \& rehabilitation, 1971;50(3):139-143.

[5]. Basmajian, J. V., \& Bazant, F. J. Factors Preventing Downward Dislocation of the Adducted Shoulder Joint An Electromyographic and Morphological Study. The Journal of Bone \& Joint Surgery,1959; 41(7):1182-1186.

[6]. Wanklyn P, Forster A, Young J. Hemiplegic shoulder pain (HSP): natural history and investigation of associated features. Disabil Rehabil.1996;18:497501.

[7]. Cailliet R. Shoulder Pain, 3rd ed. Philadelphia:F.A. Davis, 1991;2.

[8]. Park GY, Kim JM, Sohn S, Shin IH, Lee MY. Ultrasonographic Measur ement of Shoulder Subluxation in Patients with Post-stroke Hemi plegia. J Rehabil Med 2007;39:526-530.

[9]. Fugl-Meyer Assessment Upper Extremity (FMA-UE), Assessment of sensorimotor function, Rehabilitation Medicine, University of Gothenburg. Approved by Fugl-Meyer AR 2010.
[10]. Kumar, P., Cruziah, R., Bradley, M., Gray, S. and Swinkels, A. Inter-rater and intra-rater reliability of ultrasonographic measurements of acromiongreater tuberosity distance in post- stroke hemiplegia. In:Joint Summer Meeting of the British Society of Rehabilitation Medicine \& The Society for Research in Rehabilitation, Keele, UK, 4th - 5th July, 2011.

[11]. Williams, R., Taffs, L., \& Minuk, T. Evaluation of two support methods for the subluxated shoulder of hemiplegic patients. Physical Therapy, 1988;68(8):1209-1214.

[12]. Brandstater ME. Basic aspects of impairment evaluation in stroke patients. In: Chino N, Melvin JL, eds. Functional Evaluation of Stroke Patients. New York: Springer-Verlag; 1996:9-18.

[13]. Paci M, Nannetti L, Taiti P, Baccini M, Pasquini J, Rinaldi L. Shoulder subluxation after stroke: relationships with pain and motor recovery. Physiotherapy Research International. 2007 Jun1;12(2):95-104.

[14]. Zorowitz RD, Idank D, Ikai T, Hughes MB, Johnston MV. Shoulder subluxation after stroke: a comparison of four supports. Arch Phys Med Rehabil1995;76:763-71.

[15]. Zorowitz RD. Recovery patterns of shoulder subluxation nafter stroke: a six-month follow-up study. Top Stroke Rehabil.2001;8:1-9.

[16]. Kumar P, Bradley M, Gray S, Swinkels A. Association between ultrasound assessment of glenohumeral subluxation and shoulder pain, muscle strength, active range of movement and upper limb function in people with stroke. European Journal of Physiotherapy. 2019 Jan;23:1-7.

[17]. Jung KM, Choi JD. The Effects of Active Shoulder Exercise with a Sling Suspension System on Shoulder Subluxation, Proprioception, and Upper Extremity Function in Patients with Acute Stroke. Medical science monitor: international medical journal of experimental and clinical research. 2019;25:4849.

[18]. Nadler M, Pauls MM. Shoulder orthoses for the prevention and reduction of hemiplegic shoulder pain and subluxation: systematic review. Clinical rehabilitation. 2017 Apr;31(4):444-53.

[19]. Arya KN, Pandian S, Vikas, Puri V. Rehabilitation methods for reducing shoulder subluxation in poststroke hemiparesis: a systematic review. Topics in stroke rehabilitation. 2018 Jan 2;25(1):68-81.

How to cite this article:

Ushnish Mukherjee, Swapan Kumar Mishra, Prabir Mandal, Subhra Mandal, Sourav Kundu, Pankaj Kumar Mandal. SENSORI-MOTOR RECOVERY IN POST-STROKE SHOULDER SUBLUXATION: A NON INVASIVE CORRELATION STUDY. Int J Anat Res 2020;8(2.1):74187423. DOI: $10.16965 /$ ijar.2020.119 